\title{
SUPERSYMMETRIC DYNAMICS AND ZETA-FUNCTIONS
}

\author{
Nugzar Makhaldiani 1 \\ Joint Institute for Nuclear Research, Dubna
}

Boson, fermion, and super oscillators and (statistical) mechanism of cosmological constant; finite approximation of the zeta-function and fermion factorization of the bosonic statistical sum considered.

PACS: 11.30.Pb 03.65.Yz

I always knew that sooner or later $\mathrm{p}$ adic numbers will appear in Physics -

André Weil.

Supermathematics unifies discrete and continual aspects of mathematics. Boson oscillator hamiltonian is

$$
H_{b}=\hbar \omega\left(b^{+} b+b b^{+}\right) / 2=\hbar \omega\left(b^{+} b+a\right), a=1 / 2 .
$$

corresponding energy spectrum $E_{b n}$ and eigenfunctions $\mid n_{b}>$ are

$$
H_{b}\left|n_{b}>=E_{b n}\right| n_{b}>, E_{b n}=\hbar \omega\left(n_{b}+a\right), n_{b}=0,1,2, \ldots
$$

Fermion oscillator hamiltonian, eigenvectors and energies are

$$
\begin{aligned}
& H_{f}=\hbar \omega\left(f^{+} f+f f^{+}\right) / 2=\hbar \omega\left(f^{+} f-a\right), \\
& H_{f}=\left|n_{f}>=E_{f n}\right| n_{f}>, E_{f n}=\hbar \omega\left(n_{f}-a\right), n_{f}=0,1 .
\end{aligned}
$$

For supersymmetric oscillator we have

$$
\begin{aligned}
& H=H_{b}+H_{f}, H\left|n_{b}, n_{f}>=\hbar \omega\left(n_{b}+n_{f}\right)\right| n_{b}, n_{f}>, \\
& \left|n_{b}, n_{f}>=\right| n_{b}>\mid n_{f}>, E_{n_{b}, n_{f}}=\hbar \omega\left(n_{b}+n_{f}\right)
\end{aligned}
$$

For background-vacuum $\mid 0,0>$, energy $E_{0,0}=0$. For higher energy states $|n-1,1>| n,, 0>, E_{n-1,1}=E_{n, 0}$. Supersymmetry needs not only the same frequency for boson and fermion oscillators, but also that $2 a=1$.

A minimal realization of the algebra of supersymmetry

$$
\left\{Q, Q^{+}\right\}=H,\{Q, Q\}=\left\{Q^{+}, Q^{+}\right\}=0,
$$

is given by a point particle dynamics in one dimension, [1]

$$
Q=f(-i P+W) / \sqrt{2}, Q^{+}=f^{+}(i P+W) / \sqrt{2}, P=-i \partial / \partial x
$$

\footnotetext{
${ }^{1}$ e-mail address: mnv@jinr.ru
} 
where the superpotential $W(x)$ is any function of $\mathrm{x}$, and spinor operators $f$ and $f^{+}$obey the anticommuting relations

$$
\left\{f, f^{+}\right\}=1, f^{2}=\left(f^{+}\right)^{2}=0 .
$$

There is a following representation of operators $f, f^{+}$and $\sigma$ by Pauli spin matrices

$$
f=\frac{\sigma_{1}-i \sigma_{2}}{2}=\left(\begin{array}{cc}
0 & 0 \\
1 & 0
\end{array}\right), f^{+}=\frac{\sigma_{1}+i \sigma_{2}}{2}=\left(\begin{array}{cc}
0 & 1 \\
0 & 0
\end{array}\right), \sigma=\sigma_{3}=\left(\begin{array}{cc}
1 & 0 \\
0 & -1
\end{array}\right)
$$

From formulae (51) and (6) then we have

$$
H=\left(P^{2}+W^{2}+\sigma W_{x}\right) / 2 .
$$

The simplest nontrivial case of the superpotential $W=\omega x$ corresponds to the supersimmetric oscillator with Hamiltonian

$$
H=H_{b}+H_{f}, \quad H_{b}=\left(P^{2}+\omega^{2} x^{2}\right) / 2, \quad H_{f}=\omega \sigma / 2,
$$

The ground state energies of the bosonic and fermionic parts are

$$
E_{b 0}=\omega / 2, \quad E_{f 0}=-\omega / 2,
$$

so the vacuum energy of the supersymmetric oscillator is

$$
<0|H| 0>=E_{0}=E_{b 0}+E_{f 0}=0, \quad|0>=| n_{b}, n_{f}>=\left|n_{b}>\right| n_{f}>.
$$

Let us see on this toy - solution of the cosmological constant problem from the quantum statistical viewpoint. The statistical sum of the supersymmetric oscillator is

$$
Z(\beta)=Z_{b} Z_{f}
$$

where

$$
\begin{aligned}
& Z_{b}=\sum_{n} e^{-\beta E_{b n}}=e^{-\beta \omega / 2}+e^{-\beta \omega(1+1 / 2)}+\ldots=e^{-\beta \omega / 2} /\left(1-e^{-\beta \omega}\right) \\
& Z_{f}=\sum_{n} e^{-\beta E_{f n}}=e^{\beta \omega / 2}+e^{-\beta \omega / 2} .
\end{aligned}
$$

In the low temperature limit,

$$
Z(\beta)=1+O\left(e^{-\beta \omega}\right) \rightarrow 1, \quad \beta=T^{-1},
$$

so cosmological constant $\lambda \sim \ln Z \rightarrow 0$. From observable values of $\beta$ and the cosmological constant we estimate $\omega$. 
The Riemann zeta function (RZF) can be interpreted in thermodynamic terms as a statistical sum of a system with energy spectrum: $E_{n}=\ln n, n=$ $1,2, \ldots$ :

$$
\zeta(s)=\sum_{n \geq 1} n^{-s}=Z(\beta)=\sum_{n \geq 1} \exp \left(-\beta E_{n}\right), \beta=s, E_{n}=\ln n, n=1,2, \ldots
$$

Let us consider the following finite approximation of RZF

$$
\begin{aligned}
& \zeta_{N}(s)=\sum_{n=1}^{N} n^{-s}=\frac{1}{\Gamma(s)} \int_{0}^{\infty} d t t^{s-1} \frac{e^{-t}-e^{-(N+1) t}}{1-e^{-t}}=\zeta(s)-\Delta_{N}(s), \text { Re } s>1 \\
& \zeta(s)=\frac{1}{\Gamma(s)} \int_{0}^{\infty} d t \frac{t^{s-1}}{e^{t}-1}, \Delta_{N}(s)=\frac{1}{\Gamma(s)} \int_{0}^{\infty} d t \frac{t^{s-1} e^{-N t}}{e^{t}-1}
\end{aligned}
$$

Another formula, which can be used on critical line, is

$$
\begin{aligned}
\zeta(s) & =\left(1-2^{1-s}\right)^{-1} \sum_{n \geq 1}(-1)^{n+1} n^{-s} \\
& =\frac{1}{1-2^{1-s}} \frac{1}{\Gamma(s)} \int_{0}^{\infty} \frac{t^{s-1} d t}{e^{t}+1}, \text { Re } s>0
\end{aligned}
$$

Corresponding finite approximation of RZF is

$$
\begin{aligned}
& \zeta_{N}(s)=\left(1-2^{1-s}\right)^{-1} \sum_{n=1}^{N}(-1)^{n-1} n^{-s} \\
& =\frac{1}{1-2^{1-s}} \frac{1}{\Gamma(s)} \int_{0}^{\infty} \frac{t^{s-1}\left(1-\left(-e^{-t}\right)^{N}\right) d t}{e^{t}+1}=\zeta(s)-\Delta_{N}(s), \\
& \Delta_{N}(s)=\frac{1}{\Gamma(s)} \int_{0}^{\infty} d t \frac{\left.t^{s-1}\left(-e^{-t}\right)^{N}\right)}{e^{t}+1} \sim \pm N^{-s}, \\
& |s| \simeq \frac{\left|\ln \Delta_{N}(s)\right|}{\ln N}
\end{aligned}
$$

at a (nontrivial) zero of RZF, $s_{0}, \zeta_{N}\left(s_{0}\right)=-\Delta_{N}\left(s_{0}\right)$. In the integral form, dependence on $N$ is analytic and we can consider any complex valued $N$. It is interesting to see dependence (evolution) of zeros with $N$,

$$
\frac{d \Delta_{N}(s)}{d N}=(-1)^{N-1} s \Delta_{N}(s+1)
$$

For the simplest nontrivial integer $N=2$,

$$
\begin{aligned}
& \zeta_{2}(s)=\left(1-2^{1-s}\right)^{-1}\left(1-2^{-s}\right) \\
& =\frac{1-2^{-s}}{1-2^{1-s}}=\frac{2^{s}-1}{2^{s}-2}=\frac{2^{s-1 / 2}-1 / \sqrt{2}}{2^{s-1 / 2}-\sqrt{2}},
\end{aligned}
$$

we have zeros at $s=2 \pi i n / \ln 2, n=0, \pm 1, \pm 2, \ldots$ 
Let as consider the following formula (Qvelementar particles)

$$
\frac{1}{1-q}=(1+q)\left(1+q^{2}\right)\left(1+q^{4}\right) \ldots,|q|<1 .
$$

which can be proved as

$$
\begin{aligned}
& p_{k} \equiv(1+q)\left(1+q^{2}\right)\left(1+q^{4}\right) \ldots\left(1+q^{2^{k}}\right)=\frac{1-q^{2^{(k+1)}}}{1-q}, \\
& c\left(1-|q|^{2^{(k+1)}}\right)<\left|p_{k}\right|<c\left(1+|q|^{2^{(k+1)}}\right), \lim _{k \rightarrow \infty}\left|p_{k}\right|=c=1 /|1-q|, \\
& \lim _{k \rightarrow \infty} p_{k}=1 /(1-q) .
\end{aligned}
$$

The formula (22) reminds us the boson and fermion statsums

$$
Z_{b}=\frac{q^{a}}{1-q}, Z_{f}=\frac{1+q}{q^{a}}, q=\exp (-\beta \omega), a=1 / 2, \beta=1 / T
$$

and can be transformed in the following relation

$$
Z_{b}(\omega)=Z_{f}(\omega) Z_{f}(2 \omega) Z_{f}(4 \omega) \ldots
$$

Indeed,

$$
\begin{aligned}
& Z_{b}(\omega)=\frac{q^{a}}{1-q}=q^{b} Z_{f}(\omega) Z_{f}(2 \omega) Z_{f}(4 \omega) \ldots \\
& b=2 a+2 a\left(1+2+2^{2}+\ldots\right)=2 a\left(1+\frac{1}{1-2}\right)=0,|2|_{2}=1 / 2,
\end{aligned}
$$

where $|n|_{p}=1 / p^{k}, n=p^{k} m$, is p-adic norm of $n, k$ is the number of $p$ - prime factors of $n$.

Bytheway we have an extra bonus! We see that the fermion content of the boson wears the p-adic sense [2]. The prime $p=2$, in this case. Also, the vacuum energy of the oscillators wear $\mathrm{p}$ - adic sense.

We may consider also the following recurrent relations

$$
\begin{aligned}
& Z_{b}(\omega)=\frac{q^{a}}{1-q}=\frac{1+q}{q^{a}} \frac{q^{2 a}}{1-q^{2}}=Z_{f}(\omega) Z_{b}(2 \omega)=Z_{f}(\omega) Z_{f}(2 \omega) Z_{b}\left(2^{2} \omega\right)= \\
& \ldots=Z_{f}(\omega) Z_{f}(2 \omega) \ldots Z_{f}\left(2^{k-1} \omega\right) Z_{b}\left(2^{k} \omega\right)
\end{aligned}
$$

and ask that

$$
\begin{aligned}
& Z_{b}\left(2^{k} \omega\right)=1 \Rightarrow q_{k}^{2}+q_{k}-1=0 \Rightarrow q_{k}=\exp \left(-2^{k} \beta \omega\right)=\frac{\sqrt{5}-1}{2}=\frac{2}{\sqrt{5}+1} \\
& T_{k}=\beta_{k}^{-1}=2^{k} \omega / \ln g, k=0,1,2,3, \ldots, g=\frac{\sqrt{5}+1}{2}=1.618 \\
& \ln g=0.4812,1 / \ln g=2.078=2+\varepsilon, \varepsilon=0.078 \\
& T_{k}=\left(\omega_{k+1}+\varepsilon \omega_{k}\right), \omega_{k}=2^{k} \omega
\end{aligned}
$$


so, at the temperatures $T=T_{k}$, we have the finite representation of the boson statistical sum by fermion statistical sums. For higher values of $k$ and corresponding higher temperatures there are more fermion factors. We may also consider an invariant combination: $T_{k} / \omega=2^{k} / \ln g$. Note that $Z_{b}\left(\omega_{0}\right)=$ $Z_{f}\left(\omega_{0}\right), T_{0}=\omega / \ln g$. As we have seen, to $Z_{b}=1$ corresponds zero cosmological constant. It is curious to identify $T_{0}$ with relict radiation temperature.

Let us consider the simplest extension of one level fermion system,

$$
Z_{2 f}=q^{\alpha}\left(1+q+q^{2}\right), q=\exp (-\beta \omega)
$$

Now we find the vacuum energy-the parameter $\alpha$, from the following construction

$$
\begin{aligned}
& 1+q+q^{2}=\frac{1-q^{3}}{1-q} \Rightarrow q^{\alpha}\left(1+q+q^{2}\right) \frac{q^{3 / 2}}{1-q^{3}}=\frac{q^{1 / 2}}{1-q} \Downarrow \\
& Z_{2 f}(\omega) Z_{b}(3 \omega)=Z_{b}(\omega), \alpha=-1
\end{aligned}
$$

Corresponding p-state generalization is

$$
Z_{p f}(\omega) Z_{b}((p+1) \omega)=Z_{b}(\omega), Z_{p f}(\omega)=q^{-p / 2}\left(1+q+\ldots+q^{p}\right)
$$

For RZF we have the following factorized representation by prime numbers

$$
\zeta_{b}(s)=\sum_{n \geq 1} n^{-s}=\prod_{p}\left(1-p^{-s}\right)^{-1}=\left(\prod_{k} p_{k}\right)^{-a s} \prod_{p} Z_{b p}(s), Z_{b p}(s)=\frac{p^{a s}}{1-p^{-s}}
$$

it hints on boson statsums. For this we consider a fermion zeta function

$$
\begin{aligned}
& \zeta_{f}(s)=\sum_{\substack{n=p_{1}^{n_{1}} \ldots p_{k}^{n_{k}} \\
n}} n^{-s}=\prod_{p}\left(1+p^{-s}\right)=\left(\prod_{k} p_{k}\right)^{a s} \prod_{p} Z_{f p}(s), n_{k}=0,1 \\
& Z_{f p}(s)=\frac{1+p^{-s}}{p^{a s}}
\end{aligned}
$$

Now we have

$$
Z(s)=Z_{f} Z_{b}=\zeta_{f}(s) \zeta_{b}(s)=\prod_{p} \frac{1+p^{-s}}{1-p^{-s}}
$$

We may consider also the following parafermion extensions

$$
\begin{aligned}
& \zeta_{f}(s)=\sum_{m=p_{1}^{n_{1}} \ldots p_{k}^{n_{k}}} m^{-s}=\prod_{p}\left(1+p^{-s}+\ldots+p^{-s(n-1)}\right)=\left(\prod_{k} p_{k}\right)^{a s} \prod_{p} Z_{f p n}(s), \\
& Z_{f p n}(s)=\frac{1+p^{-s}+\ldots+p^{-s(n-1)}}{p^{a s}}, n_{k}=0,1, \ldots, n-1 \\
& Z(s)=Z_{f} Z_{b}=\zeta_{f p n}(s) \zeta_{b}(s)=\prod_{p} \frac{1+p^{-s}+\ldots+p^{-s(n-1)}}{1-p^{-s}}
\end{aligned}
$$


A braid group $B_{N+1}$ is generated by elements $s_{n}, n=1, \ldots N$, subject to relations:

$$
s_{n} s_{n+1} s_{n}=s_{n+1} s_{n} s_{n+1}, s_{n} s_{m}=s_{m} s_{n}, m \neq n \pm 1
$$

An A-Type Hecke algebra $H_{N+1}=H_{N+1}(q)$ (see, e.g., [3] and references therein) is a quotient of the group algebra of the braid group $B_{N+1}$ by a Hecke relations

$$
s_{n}^{2}=1+\lambda s_{n}, n=1, \ldots N, \lambda=q-q^{-1},
$$

where $q \in C /\{0\}$ is a parameter. Note that, for $\lambda=-1$,

$$
\begin{aligned}
& q^{2}+q-1=0, \\
& s_{n}^{2}+s_{n}-1=0 .
\end{aligned}
$$

As another example where similar structure appears, let us consider, on the base of familiar sequence, the following discrete dynamical system

$$
f_{n+1}=f_{n}+f_{n-1}
$$

which reduce when $f_{n} \neq 0$ to the following one

$$
\varphi_{n+1}=1+1 / \varphi_{n}, \varphi_{n}=f_{n} / f_{n-1}
$$

with convergent points $x_{ \pm}$:

$$
\begin{aligned}
& x_{+}=1+\frac{1}{1+x_{+}}=1+\frac{1}{1+\frac{1}{1+x_{+}}}=\ldots=1+\frac{1}{1+\frac{1}{1+\ldots}}=\frac{\sqrt{5}-1}{2}, \\
& x_{-}=-1-x_{+}=-1+\frac{1}{x_{-}}=-1+\frac{1}{-1+\frac{1}{-1+\ldots}}=-\frac{\sqrt{5}+1}{2}
\end{aligned}
$$

We may consider this dynamical system as exactly solvable example of one charge renormdynamics with ultraviolet and infrared fixed points as $x_{-}$and $x_{+}$.

It is not obvious that the dynamical system (40) is reversible: knowing $f_{n+1}$ we can not define $f_{n}$. On the level of pares $\left(f_{n}, f_{n-1}\right)$ we obtain explicitly reversible system:

$$
F_{n+1}=A F_{n-1}, F_{n}=\left(\begin{array}{c}
f_{n} \\
f_{n-1}
\end{array}\right), A=\left(\begin{array}{cc}
2 & 1 \\
1 & 1
\end{array}\right), \operatorname{det} A=1 .
$$

So, the formulation with explicit time reflection symmetry needs one, the simplest, step of renormdynamics: double time steps. On this level appears internal degrees of freedom-spin. 


\section{Список литературы}

[1] Witten E.// Nucl. Phys. B. 1981. V. 188. P. 513.

[2] Makhaldiani N.// Fractal Calculus (H) and some Applications, Physics of Particles and Nuclei Letters. 2011. V. 8. P. 325.

[3] Wenzl H.// Hecke algebras of type An and subfactors, Invent. Math. 1988. V. 92. P. 349. 